\title{
Density-Controlled Growth of Aligned ZnO Nanowires Sharing a Common Contact: A Simple, Low-Cost, and Mask-Free Technique for Large-Scale Applications
}

\author{
Xudong Wang, ${ }^{\dagger}$ Jinhui Song, ${ }^{\dagger}$ Christopher J. Summers,${ }^{\dagger}$ Jae Hyun Ryou, ${ }^{\ddagger}$ Peng Li, \\ Russell D. Dupuis, and Zhong L. Wang*, \\ School of Materials Science and Engineering and School of Electrical and Computer Engineering, Georgia \\ Institute of Technology, Atlanta, Georgia 30332
}

Received: January 17, 2006; In Final Form: March 1, 2006

\begin{abstract}
An effective, low cost, simple, and mask-free pathway is demonstrated for achieving density control of the aligned $\mathrm{ZnO}$ nanowires grown for large-scale applications. By a slight variation of the thickness of the thermally evaporated gold catalyst film, a significant change in the density of aligned $\mathrm{ZnO}$ nanowires has been controlled. The growth processes of the nanowires on an $\mathrm{Al}_{0.5} \mathrm{Ga}_{0.5} \mathrm{~N}$ substrate has been studied based on the wetting behavior of gold catalyst with or without source vapor, and the results classify the growth processes into three categories: separated dots initiated growth, continuous layer initiated growth, and scattered particle initiated growth. This study presents an approach for growing aligned nanowire arrays on a ceramic substrate with the simultaneous formation of a continuous conducting electrode at the roots, which is important for device applications, such as field emission.
\end{abstract}

One-dimensional (1D) $\mathrm{ZnO}$ nanostructures are considered to be one of the most important semiconducting nanomaterials for fabricating nanodevices with applications in optics, electronics, mechanics, and biomedical sciences. ${ }^{1,2}$ The attraction of $\mathrm{ZnO}$ is the result of its high purity, high crystallinity, wide direct energy band gap (3.37 eV), large excitation binding energy (60 $\mathrm{meV})$, piezoelectricity, and biocompatibility, as well as the divisive nanostructures. ${ }^{3}$ Since the successful growth of aligned $\mathrm{ZnO}$ nanowires on a single-crystal substrate, ${ }^{4-6}$ a system that may be very useful for vertical device fabrication has been found. As a result, great interest in acquiring more control over the alignments, including supporting substrates, distribution of nanowires, and density of nanowires, to maximally meet the requirements of nanodevices has been inspired.

The vapor-liquid-solid (VLS) process is the most widely used technique for growing nanowires because of its relatively low cost and simple procedure. ${ }^{4-8}$ Metal-organic chemical vapor deposition (MOCVD) has also been proven as an alternative method for aligned nanowires but with a much higher cost. ${ }^{9,10}$ A wet chemistry process has recently been demonstrated as a powerful technique for growing aligned nanowires at a very low cost and over a very large surface area. ${ }^{11-13}$ However, the density of nanowires grown on the surface still cannot be controlled unless a catalyst pattern created by a mask or lithography is applied.

From the application point of view, the density of the aligned nanowires is very important since it is directly related to how the nanowires interact with each other optically, electronically, and mechanically. In field emission, ${ }^{14,15}$ for example, an array of densely packed nanowires greatly reduces the field enhancement effect at the nanowire tip to a level not much different from a flat metal plate, while too loosely distributed nanowires cannot meet the desired requirement of high-emitting points. The ability to systematically control the density of the aligned

* Corresponding author. E-mail: zhong.wang@mse.gatech.edu.

$\dagger$ School of Materials Science and Engineering.

$\leftarrow$ School of Electrical and Computer Engineering. nanowires so that optimal performance can be achieved by adjusting the space between the aligned nanowires when their morphologies already meet the requirements is strongly desired. So far, two methods are used in tuning the density. One method is to adjust the concentration of catalyst in a colloidal solution before applying it onto the substrate, ${ }^{14}$ but the uniformity of nanoparticle distributions over large surface areas is questionable because of the agglomeration of the particles during the drying process. Another method is to use a patterning technique, ${ }^{16}$ such as mask, lithography, or scanning probe microscopy, which can provide precise control over the density but at a much higher cost. Also the speed cannot meet the requirement of large-area growth.

After a systematic study of the growth of $\mathrm{ZnO}$ nanowires on nitride substrates, ${ }^{17,18}$ in this paper, we investigated the effects of the thickness of the gold catalyst layer. ${ }^{19}$ It's generally believed that in the VLS process, the size of the catalyst particles determines the width of nanowires. However, in our experiments, we found that this rule only applies when the catalyst particles are reasonably small $(<40 \mathrm{~nm})$. Because of the wetting situation between the melted catalyst droplet and the substrate, more energy favorable sites were created for nanowire growth with thinner catalyst layers. As a result, varying the thickness of the deposited gold film can be a simple but effective pathway for controlling the density of aligned $\mathrm{ZnO}$ nanowires. Moreover, when the catalyst layer was sufficiently thick, a continuous $\mathrm{ZnO}$ network would be deposited simultaneously at the bottom of the nanowires. This layer may serve as the electrode for many electrical and device applications. The density-dependent PL property of the nanowires grown has also been studied.

The $\mathrm{Al}_{0.5} \mathrm{Ga}_{0.5} \mathrm{~N}$ substrate was fabricated by the MOCVD technique. ${ }^{20}$ The $c$-plane-orientated $\mathrm{Al}_{0.5} \mathrm{Ga}_{0.5} \mathrm{~N}$ epitaxial layer with a thickness of $\sim 205 \mathrm{~nm}$ was grown on a $500 \mathrm{~nm}$ thick AlN buffer layer which was grown on a one-side-polished $c$-plane-oriented sapphire substrate. Eight stripes of gold layers with thicknesses from 1 to $8 \mathrm{~nm}$ at $1 \mathrm{~nm}$ gradient were deposited onto a $2 \times 1 \mathrm{~cm} \mathrm{Al}_{0.5} \mathrm{Ga}_{0.5} \mathrm{~N}$ substrate using a gold thermal 


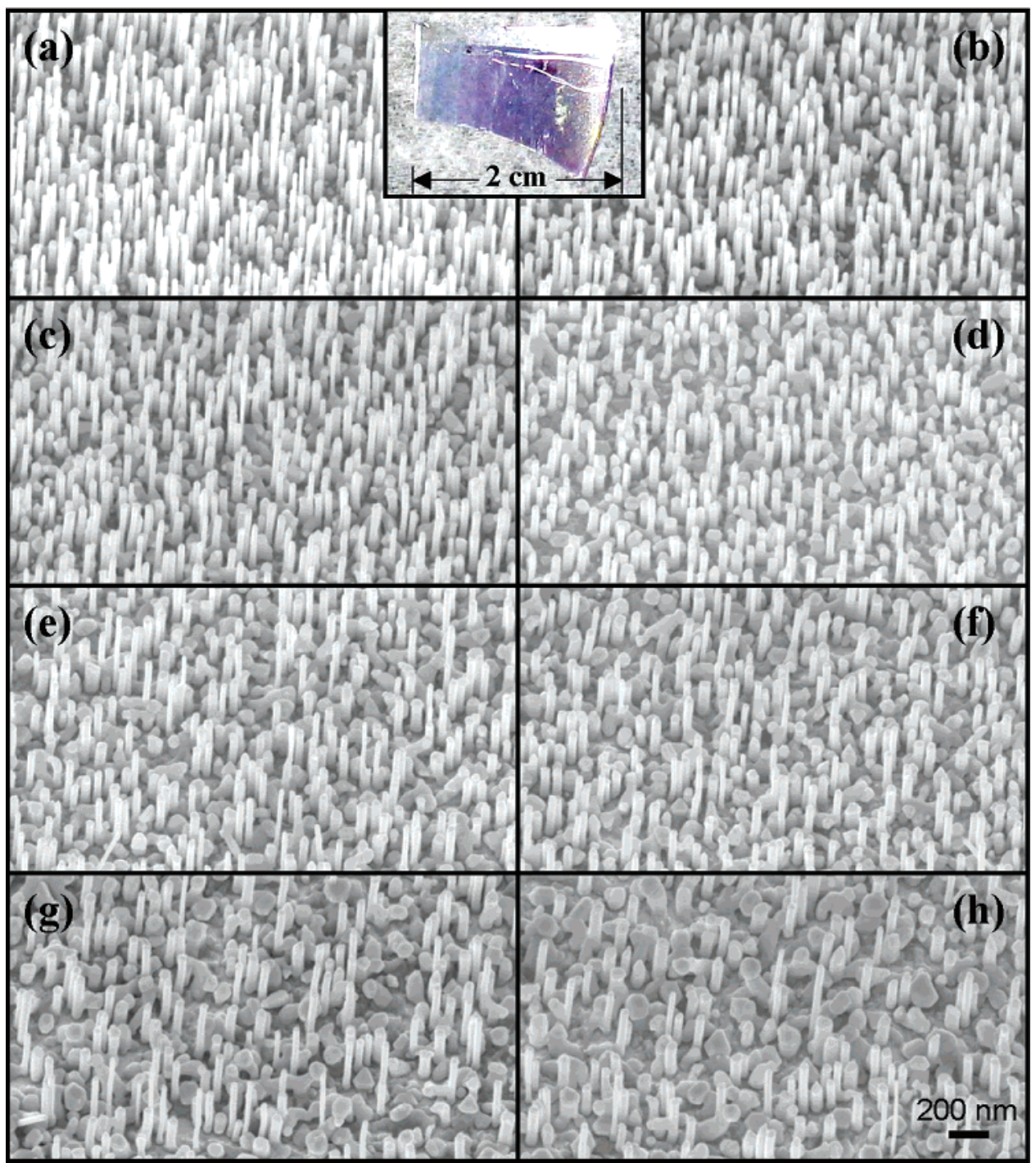

Figure 1. $(\mathrm{a}-\mathrm{h}) \mathrm{SEM}$ images of the aligned $\mathrm{ZnO}$ nanowires grown on a nitride substrate as catalyzed by a deposited gold film of thicknesses varying from 1 to $8 \mathrm{~nm}$ in $1 \mathrm{~nm}$ steps. Inset: Optic photo of the substrate showing eight stripes of the aligned ZnO nanowires from left to right corresponding to images $\mathrm{a}-\mathrm{h}$.

evaporator, where the thickness was measured by a quartz crystal thickness monitor with a resolution of $0.1 \mathrm{~nm}$. The fabrication of $\mathrm{ZnO}$ nanowires was carried out under the optimal experimental conditions we developed previously. ${ }^{18}$ In general, a mixture of equal amounts (by weight) of $\mathrm{ZnO}$ and graphite powders were used as source materials and were loaded into an alumina boat, which was located at the center of an alumina tube. To facilitate the reaction, $2 \%(1 \mathrm{sccm})$ oxygen was mixed with argon carrier gas at a flow rate of $49 \mathrm{sccm}$, and the substrates were placed down stream in a temperature zone of $\sim 850{ }^{\circ} \mathrm{C}$. A horizontal tube furnace was used to heat the source materials to $950{ }^{\circ} \mathrm{C}$ at a rate of $50{ }^{\circ} \mathrm{C} / \mathrm{min}$, and the temperature was held at the peak temperature for $30 \mathrm{~min}$ under a pressure of 30 mbar. The system was slowly cooled to room temperature under flowing argon gas.

To investigate the effects of the thickness of the Au catalyst on the density of the nanowires grown, eight stripes of gold layers with thicknesses from 1 to $8 \mathrm{~nm}$ with a $1 \mathrm{~nm}$ gradient were employed as catalysts. Through a thermal evaporation process, all of the nanowires were grown simultaneously and under identical experimental conditions on gold films with different thickness. The inset in Figure 1 is an optical picture of the substrate, from which the eight stripes can be clearly distinguished. From right to left, the color of the stripes varies from light blue to purple, which represent the aligned $\mathrm{ZnO}$ nanowires catalyzed by Au layers of thicknesses from 1 to 8 $\mathrm{nm}$. Each stripe of as-synthesized $\mathrm{ZnO}$ nanowires exhibited distinct appearances even though there was only $1 \mathrm{~nm}$ change in the thickness of the deposited Au film. Since the blue color is from $\mathrm{ZnO}$ and red color is contributed by gold, it can be assumed the thinner layer of the gold catalyst resulted in more $\mathrm{ZnO}$ nanowires, which has been further confirmed by scanning electronic microscopy (SEM). Each stripe of the as-synthesized $\mathrm{ZnO}$ nanowires exhibited obvious differences even though there was only $1 \mathrm{~nm}$ change of thickness between two adjacent stripes.

Figure $1 \mathrm{a}-\mathrm{h}$ represents typical SEM images recorded from the nanowires grown in the areas of the eight stripes at an identical magnification. Clearly, the $1 \mathrm{~nm}$ gold layer gives the highest density of aligned $\mathrm{ZnO}$ nanowires (Figure 1a), while the thickest gold layer results in the lowest density (Figure 1h). With the decrease of the density of the $\mathrm{ZnO}$ nanowires, more and more larger particles were observed on the substrate. As confirmed by energy-dispersive X-ray spectroscopy (EDS) analysis equipped on the SEM, those particles are mostly composed of gold, which makes the substrate appear more reddish in the optical image. However, despite the large difference in density, all of the $\mathrm{ZnO}$ nanowires exhibit very similar width and length distributions. 

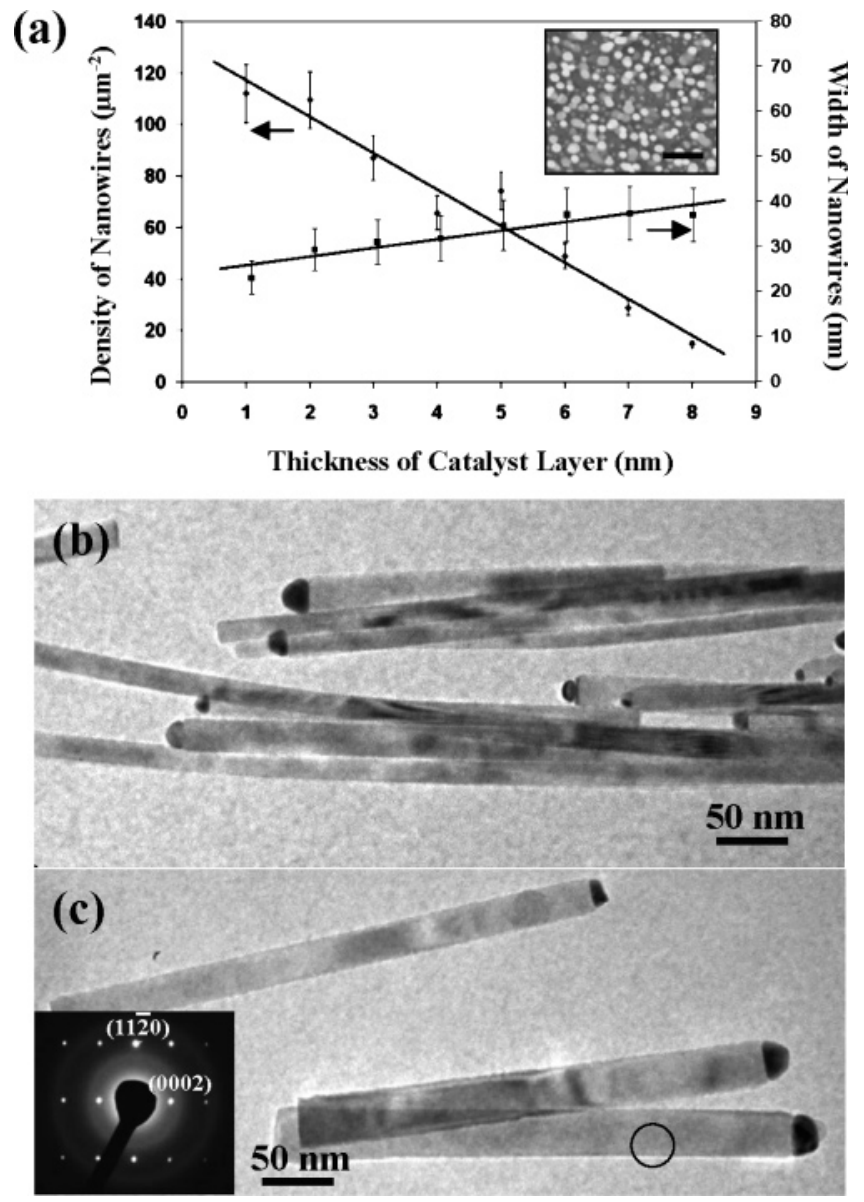

Figure 2. (a) Variation of density (left-hand vertical axis) and width (right-hand vertical axis) of the aligned $\mathrm{ZnO}$ nanowires with the thickness of gold catalyst layer. Inset: Top-view SEM image of the aligned $\mathrm{ZnO}$ nanowires used for density calculation, the scale bar represents $200 \mathrm{~nm}$. (b, c) TEM images of $\mathrm{ZnO}$ nanowires catalyzed by 1 and $8 \mathrm{~nm}$ gold layers, respectively. Inset: Selected area electron diffraction pattern recorded from a nanowire indicated by the circle in image $\mathrm{c}$.

Quantitative analyses were thereafter performed to reveal the density and thickness relationships of the aligned nanowires. The density was measured by top-view SEM images, as shown in the inset of Figure 2a. Each bright spot corresponds to a perpendicular $\mathrm{ZnO}$ nanowire with a gold catalyst particle on top. By counting the number of bright dots on five randomly taken SEM images, the average number of nanowires per square micrometer was calculated. The nanowire density at each thickness of gold catalyst layer is shown on the left-hand side axis in Figure $2 \mathrm{a}$ with an averaged error of $\sim 10 \%$. An almost linear drop in density from 112 to 15 with increasing catalyst thickness can be observed. The width of the nanowires was measured by transmission electronic microscopy (TEM). After the aligned nanowires on each stripe were transferred to different TEM grids, more than 10 TEM images were taken for each sample to calculate the average width. The result is shown in Figure $2 \mathrm{a}$. The standard deviations of the samples are $\sim 16 \%$ from $>100$ randomly chosen nanowires. Unlike the density, the average width remains between 30 and $40 \mathrm{~nm}$ despite the change in catalyst thickness. To present the variation in nanowire width, representative TEM images with the same magnification for the two extreme cases, nanowires catalyzed by $1 \mathrm{~nm}$ and $8 \mathrm{~nm}$ thick gold layers, were taken and are shown in Figure $2 b$ and $c$, respectively. One nanowire in Figure 2c exhibits a similar width as that in Figure 2b, while the other two are about $10-15 \mathrm{~nm}$ wide, indicating a larger absolute width distribution for $8 \mathrm{~nm}$
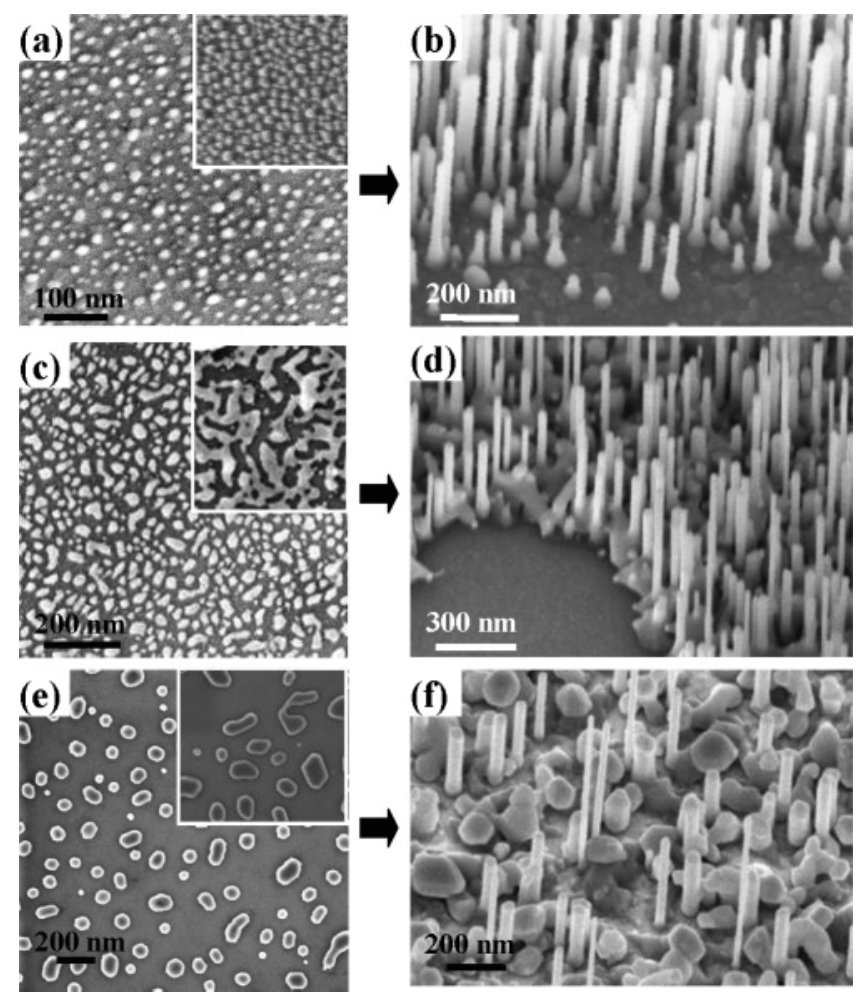

Figure 3. Three categories of the growth processes based on the thickness of gold layer. (a, c, e) SEM images showing the wetting situation of gold catalyst on $\mathrm{Al}_{0.5} \mathrm{Ga}_{0.5} \mathrm{~N}$ substrate prior to the nucleation of the nanowires. Insets: SEM images with the same magnification showing the wetting situation when the vapor source was presented to initiate the growth. (b, d, f) SEM images of the aligned $\mathrm{ZnO}$ nanowires catalyzed by the corresponding gold layers.

thick gold layer catalyzed nanowires. A selective-area electron diffraction pattern was taken on the nanowire as indicated by a circle in Figure 2c. As shown in the inset of Figure 2c, the nanowires were grown along the [0001] direction, which is the fastest growth direction for the VLS growth of $\mathrm{ZnO}$.

Because of the linear relationship between the density of the nanowires and the thickness of the catalyst layer, thickness control of gold catalyst could be a very simple and effective way to achieve density control of aligned nanowires over a large surface area. To reveal why the density varies but the width remains constant, the wetting behavior of a gold layer on the $\mathrm{Al}_{0.5} \mathrm{Ga}_{0.5} \mathrm{~N}$ substrate was investigated when heated to the growth temperature. Two additional pieces of $\mathrm{Al}_{0.5} \mathrm{Ga}_{0.5} \mathrm{~N}$ substrates with the same gold catalyst distribution were prepared. Under the same furnace growth condition, one piece of the substrate was heated to the growth temperature without introducing source material to reveal the formation of catalyst dots; while the other one was heated to the same temperature with source material in the growth chamber for only a few minutes to reveal the initial growth stage of the nanowires. On the basis of the data we have received, the growth situation can be divided into three categories, as shown in Figure 3.

The first category is the separated dots initiated growth, where the gold layer is only $1-2 \mathrm{~nm}$ in thickness. As shown in Figure $3 \mathrm{a}$, after it was heated to the growth condition, the 1-2 $\mathrm{nm}$ gold layer melted into tiny gold dots $(10-20 \mathrm{~nm})$ evenly distributed on the substrate surface. Once the source vapor was present, larger particles were observed because of the coarsening effect resulting from the formation of a supersaturated "alloy", as shown in the inset of Figure 3a. However, all of the particles 
(a)

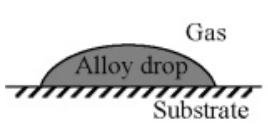

Stage 1 pre-initiation (b)

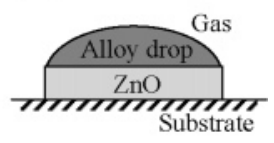

Stage 2 post-initiation
Figure 4. Schematic of (a) pre- and (b) post-initiation of $\mathrm{ZnO}$ nanowire growth.

remained separated from each other, resulting in a higher density and individual $\mathrm{ZnO}$ nanowire growth from the substrate surface (Figure 3b).

The second category is continuous layer initiated growth when the thickness of the gold deposition layer was 3-6 nm. As shown in Figure 3c, the gold layer melted into separated islands with different sizes at the deposition temperature. Most of the large islands then merge into a continuous network on the substrate surface when alloying gold with the source vapor at the initial stage (inset of Figure 3c), while the small islands remained isolated. As the deposition continued, the isolated small islands initiated the growth of nanowires, while the network formed a complete layer covering the substrate and connecting the base of all the nanowires (Figure 3d). The gold in the network would eventually be precipitated out forming small dots or very short rods during the cooling process. Therefore, the density of nanowires that can be grown in this case is relatively lower. However, since every single $\mathrm{ZnO}$ nanowire is interconnected by the $\mathrm{Au}$ or $\mathrm{ZnO}$ layer at the root, this can be an alternative pathway to electrically connecting the aligned $\mathrm{ZnO}$ nanowires for electronic nanodevices and field emission. This is a unique advantage for solving the bottom electrical contact problem. It is also has the great merit of replacing the expensive semiconducting substrates by sapphire, while preserving the electrical contact, offering great potential for industrial application.

The third category is called the scattered islands initiated growth; this occurs when the thickness of the gold layer is greater than $7 \mathrm{~nm}$. In this case, when melted, the catalyst layer is thick enough to form big gold particles $(\sim 100 \mathrm{~nm})$. The large gold particles were widely separated; also a very small number of gold dots were left, as shown in Figure 3e. Even after an alloy was formed with the source vapor, this morphology remained unchanged (inset of Figure 3e). In the deposition process, only the small dots could initiate the growth of $\mathrm{ZnO}$ nanowires; and the big particles led the growth of $\mathrm{ZnO}$ films/ junks lying on the substrate, resulting in the lowest density of aligned $\mathrm{ZnO}$ nanowires.

The above discussion is based on moderate growth conditions as described earlier, under which the evaporation of source materials was relatively slow so that the catalyst layer had enough time to melt and form an alloy step by step, which can be considered as a thermal equilibrium process. To explain why only the small dots initiate growth, the thermodynamic mechanisms were studied based on the pre- and postnucleation of the $\mathrm{ZnO}$ nanowire, as illustrated in Figure 4. The alloy droplet is assumed to be supersaturated at stage 1; stage 2 presents the moment when the first layer of $\mathrm{ZnO}$ is precipitated, during which the change in supersaturation is compensated by the diffusing of source vapor from the gas phase into the alloy. The free energies of these two stages are presented by $G_{1}$ and $G_{2}$, respectively, as

$$
\begin{array}{r}
G_{1}=V_{\mathrm{A}} G_{\mathrm{V} 1}^{\mathrm{A}}+V_{\mathrm{G}} G_{\mathrm{V} 1}^{\mathrm{G}}+A_{\mathrm{AS}} \gamma_{\mathrm{AS}}+A_{\mathrm{AG}} \gamma_{\mathrm{AG}}+A_{\mathrm{GS}} \gamma_{\mathrm{GS}} \\
G_{2}=V_{\mathrm{A}} G_{\mathrm{V} 2}^{\mathrm{A}}+V_{\mathrm{G}} G_{\mathrm{V} 2}^{\mathrm{G}}+A_{\mathrm{AZ}} \gamma_{\mathrm{AZ}}+A_{\mathrm{ZS}} \gamma_{\mathrm{ZS}}+A_{\mathrm{ZG}} \gamma_{\mathrm{ZG}}+ \\
A_{\mathrm{AG}} \gamma_{\mathrm{AG}}+A_{\mathrm{GS}} \gamma_{\mathrm{GS}}+V_{\mathrm{Z}} G_{\mathrm{V}}^{\mathrm{Z}}
\end{array}
$$

where $V_{\mathrm{A}}, V_{\mathrm{G}}$, and $V_{\mathrm{Z}}$ are the volume of the alloy droplet, gas phase, and $\mathrm{ZnO}$, respectively, $G_{\mathrm{V} 1}^{\mathrm{A}}, G_{\mathrm{V} 1}^{\mathrm{G}}$ and $G_{\mathrm{V} 2}^{\mathrm{A}}, G_{\mathrm{V} 2}^{\mathrm{G}}$ are the free energy per unit volume of alloy and gas phase at the first and second stages, respectively, $G_{\mathrm{V}}^{\mathrm{Z}}$ is the free energy per unit volume of the precipitated $\mathrm{ZnO}$ crystal, and $A$ and $\gamma$ are the area and free energy of the interfaces, respectively, between gas, alloy, substrate, and $\mathrm{ZnO}$ surfaces as indicated by their initials in the subscriptions. The formation of $\mathrm{ZnO}$ results in a free energy change $\Delta G=G_{2}-G_{1}$. By assuming the alloy composition remains the same $\left(G_{\mathrm{V} 1}^{\mathrm{G}}=G_{\mathrm{V} 2}^{\mathrm{G}}\right)$ and the area of precipitated $\mathrm{ZnO}$ is the same as the contact area between the alloy and the substrate $\left(A_{\mathrm{AS}}=A_{\mathrm{AZ}}=A_{\mathrm{ZS}}\right)$, the $\Delta G$ can be given by

$$
\begin{aligned}
\Delta G=V_{\mathrm{G}}\left(G_{\mathrm{V} 2}^{\mathrm{G}}-G_{\mathrm{V} 1}^{\mathrm{G}}\right)+A_{\mathrm{AS}}\left(\gamma_{\mathrm{AZ}}+\gamma_{\mathrm{ZS}}-\gamma_{\mathrm{AS}}\right)+ \\
A_{\mathrm{ZG}} \gamma_{\mathrm{ZG}}+V_{\mathrm{Z}} G_{\mathrm{V}}^{\mathrm{Z}}
\end{aligned}
$$

To initiate the precipitation of $\mathrm{ZnO}, \Delta G$ has to be negative, so we have

$$
\begin{array}{r}
V_{\mathrm{G}}\left(G_{\mathrm{V} 1}^{\mathrm{G}}-G_{\mathrm{V} 2}^{\mathrm{G}}\right)>A_{\mathrm{AS}}\left(\gamma_{\mathrm{AZ}}+\gamma_{\mathrm{ZS}}-\gamma_{\mathrm{AS}}\right)+A_{\mathrm{ZG}} \gamma_{\mathrm{ZG}}+ \\
V_{\mathrm{Z}} G_{\mathrm{V}}^{\mathrm{Z}}
\end{array}
$$

The left-hand side of the inequality is the change in the free energy of the vapor phase; on the right-hand side, the first term is determined by the initial size of the catalyst, $A_{\mathrm{AS}}$, and last two terms are negligible at the initiation nucleation stage since the size of the $\mathrm{ZnO} /$ gas interface, $A_{\mathrm{ZG}}$, and the volume of $\mathrm{ZnO}$, $V_{\mathrm{Z}}$, are very small. So eq 4 can be simplified to

$$
G_{\mathrm{V} 1}^{\mathrm{G}}-G_{\mathrm{V} 2}^{\mathrm{G}}>C A_{\mathrm{AS}}, C=\left(\gamma_{\mathrm{AZ}}+\gamma_{\mathrm{ZS}}-\gamma_{\mathrm{AS}}\right) / V_{\mathrm{G}}
$$

where $C$ is a constant. From this equation, small $A_{\mathrm{AS}}$ values (e.g., small gold dots) are thermodynamically favorable sites for the initiation of aligned $\mathrm{ZnO}$ nanowires. Since the surface area, $A_{\mathrm{AS}}$, for small size gold particles is rather small, eq 5 is usually satisfied. Therefore, growth on small size gold particles is thermodynamically favorable.

On the other hand, small gold particles are very likely to be in the liquid state at the growth temperature because of the reduced melting point for smaller particles. From the experiments of Buffat, ${ }^{21}$ the melting point of gold layer below $5 \mathrm{~nm}$ is much lower $\left(<800^{\circ} \mathrm{C}\right)$ than the melting temperature of bulk gold $\left(1064{ }^{\circ} \mathrm{C}\right)$. These molten small gold particles resulted in the nucleation and growth of aligned $\mathrm{ZnO}$ nanowires resulting from the VLS process, while the large gold islands are most likely in the solid-state, thus growth by the VLS process is not possible. In our experiments, most of the nanowires are around $30-40 \mathrm{~nm}$, and therefore, we may disregard the deposition thickness of the gold film.

Photoluminescence (PL) measurements were performed on the samples to reveal the light-emitting properties of the aligned $\mathrm{ZnO}$ nanowires with different densities. Figure 5 shows eight PL curves of the aligned $\mathrm{ZnO}$ nanowires catalyzed by gold layers with thicknesses from 1 to $8 \mathrm{~nm}$. Strong luminescence peaks are observed at $377 \mathrm{~nm}$ from all of the eight curves, while the intensities are varied. This is consistent with the constant diameter of the nanowires for all of the samples. In general, 


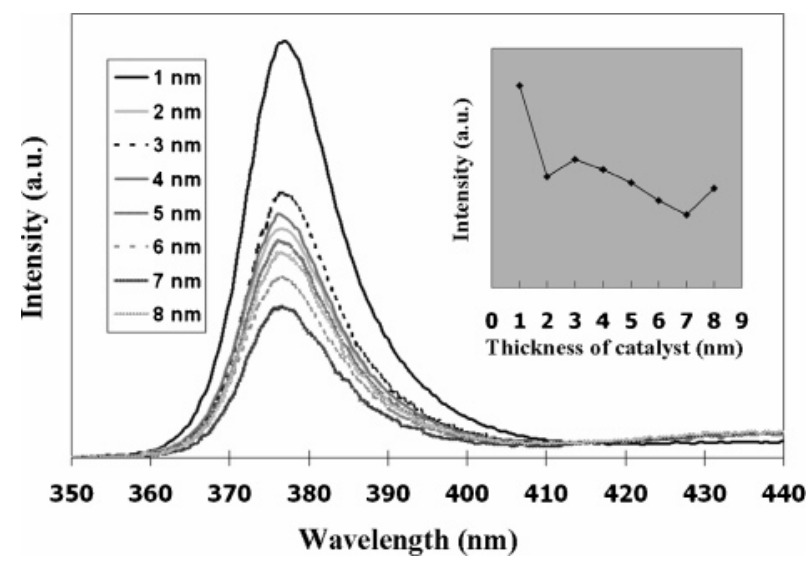

Figure 5. Photoluminescence spectra of the eight stripes of the aligned $\mathrm{ZnO}$ nanowires catalyzed by gold layers of thicknesses from 1 to 8 $\mathrm{nm}$ in $1 \mathrm{~nm}$ steps. Inset: Variation of the intensity of PL peaks with the change of catalyst thickness.

the intensity decreased with lower density, as shown in the inset of Figure 5. The highest PL intensity was from the nanowires catalyzed by a $1 \mathrm{~nm}$ gold layer, which has the highest density. However, the lowest PL intensity was not from the sample with the lowest density. On the contrary, the sample catalyzed by an $8 \mathrm{~nm}$ gold layer shows an enhanced PL intensity. This is probably because of the increased reflectivity of the emitted light by the large gold particles lying on the substrate. The reflectance from samples 2 and 3 may also be attributed to the increased reflectivity resulting from the formation of a continuous $\mathrm{ZnO}$ bottom layer.

In summary, we have found that a slight variation of the thickness of gold catalyst results in a significant change in the density of aligned $\mathrm{ZnO}$ nanowiresgrown. This could be an effective and simple pathway for the control of nanowire density without using sophisticated techniques such as catalyst patterning. The growth processes of $\mathrm{ZnO}$ nanowires on the $\mathrm{Al}_{0.5} \mathrm{Ga}_{0.5} \mathrm{~N}$ substrate has been studied based on the wetting behavior of gold catalyst on the substrate with or without source vapor, and the results classify the growth into three categories: separated dots initiated growth, continuous layer initiated growth, and scattered particle initiated growth. This study demonstrates two remarkable results: growth of aligned nanowire arrays on a ceramic substrate with simultaneous formation of a continuous conducting electrode at the roots and an easy and effective way for controlling the density of nanowires grown on large surfaces for industrial applications. These results are important for device applications.

Acknowledgment. The research was partially supported by the DARPA SUVOS program, NASA Vehicle Systems Program and Department of Defense Research and Engineering (DDR\&E), and MURI program from ARO: Grant DAAD19-01-1-0603. In addition, R.D.D. gratefully acknowledges the support of the Steve W. Chaddick Endowed Chair in Electrooptics.

\section{References and Notes}

(1) Wang, Z. L. J. Phys.: Condens. Matter 2004, 16, R829.

(2) Wang, Z. L. Mater. Today 2004, 7, 26.

(3) Özgür, Ü.; Alivov, Y. I.; Liu, C.; Teke, A.; Reshchikov, M. A.; Do"'an, S.; Avrutin, V.; Cho, S.-J.; Morkoç, H. J. Appl. Phys. 2005, 98, 041301.

(4) Huang, M.; Mao, S.; Feick, H.; Yan, H.; Wu, Y.; Kind, H.; Weber, E.; Russo, R.; Yang, P. Science 2001, 292, 1897.

(5) Zhao, Q. X.; Willander, M.; Morjan, R. E.; Hu, Q. H.; Campbell, E. E. E. Appl. Phys. Lett. 2003, 83, 165. 423.

(6) Wang, X. D.; Summers, C. J.; Wang, Z. L. Nano Lett. 2004, 4,

(7) Duan, X.; Lieber, C. M. Adv. Mater. 2000, 12, 298. 2066.

(8) Ding, Y.; Gao, P. X.; Wang, Z. L. J. Am. Chem. Soc. 2004, 106

(9) Martensson, T.; Svensson, C. P. T.; Wacaser, B. A.; Larsson, M. W.; Seifert, W.; Deppert, K.; Gustafsson, A.; Wallenberg, L. R.; Samuelson, L. Nano Lett. 2004, 4, 1987.

(10) Park, W. I.; Kim, D. H.; Jung, S. W.; Yi, G. C. Appl. Phys. Lett. 2002, 80,4232 .

(11) Greene, L.; Law, M.; Tan, D. H.; Goldberger, J.; Yang, P. Nano Lett. 2005, 5, 1231.

(12) Hsu, J. W. P.; Tian, Z. R.; Simmons, N. C.; Matzke, C. M.; Voigt, J. A.; Liu, J. Nano Lett 2005, 5, 83.

(13) Tian, Z. R.; Voigt, J. A.; Liu, J.; Mckenzie, B.; McDermott, M. J.; Rodriguez, M. A.; Konishi, H.; Xu, H. Nature Mater. 2003, 2, 821.

(14) Jo, S. H.; Tu, Y.; Huang, Z. P.; Carnahan, D. L.; Wang, D. Z.; Ren, Z. F. Appl. Phys. Lett. 2003, 82, 3520.

(15) Nilsson, L.; Groening, O.; Emmenegger, C.; Kuettel, O.; Schaller, E.; Schlapbach, L.; Kind, H.; Bonard, J. M.; Kern, K. Appl. Phys. Lett. 2000, 76, 2071.

(16) Martensson, T.; Carlberg, P.; Borgstrom, M.; Montelius, L.; Seifert, W.; Samuelson, L. Nano Lett. 2004, 4, 699.

(17) Wang, X. D.; Song, J. H.; Li, P.; Ryou, J. H.; Dupius, R. D.; Summers, C. J.; Wang, Z. L. J. Am. Chem. Soc. 2005, 127, 7920.

(18) Song, J. H.; Wang, X. D.; Riedo, E.; Wang, Z. L. J. Phys. Chem. B 2005, 109, 9869.

(19) Cui, Y.; Lauhon, L. J.; Gudiksen, M. S. Appl. Phys. Lett. 2001, $78,2214$.

(20) Zhu, T. G.; Chowdhury, U.; Denyszyn, J. C.; Wong, M. M.; Dupuis, R. D. J. Cryst. Growth 2003, 248, 548.

(21) Buffat, P.; Borel, J. P. Phys. Rev. A 1976, 13, 2287. 